\title{
Effective interventions to improve long- term physiotherapy exercise adherence among patients with lower limb osteoarthritis. A systematic review
}

\author{
Pathmanathan Cinthuja ${ }^{* *}$, Nidhya Krishnamoorthy ${ }^{2}$ and Gamalendira Shivapatham ${ }^{3}$
}

\begin{abstract}
Introduction: Osteoarthritis $(\mathrm{OA})$ is a chronic condition. Physiotherapy is known to be beneficial for people with $O A$. Patient adherence to physiotherapy exercise is essential for the effective management of OA.

Objectives: To determine different methods used to enhance physiotherapy exercise adherence for a period of more than 12 months among patients with $\mathrm{OA}$ and to report the most effective methods to enhance exercise adherence among people with lower limb OA.

Design: Systematic review.

Methods: PubMed, Pedro, Web of Science, and EMBASE databases were searched for randomized controlled trials, cohort studies, case-control studies, and cross-sectional studies published in the English language from 2000 to 2020. The literature search was done on 27 August 2020. Two researchers independently conducted the screening, eligibility assessment, data extraction, methodology quality assessment using the PEDro scale, and risk of bias assessment using RoB2. A narrative synthesis of key outcomes is presented, percentage of adherence rate; Preferred Reporting Items for Systematic Review was used to report the review. Meta-analysis was not performed due to heterogeneity of studies. The study protocol was registered in Prospero (Prospero ID: CRD42020205653).

Results: The primary search strategy identified 5839 potentially relevant articles, of which 5157 remained after discarding duplicates. After screening based on title and abstract, 40 papers were potentially eligible for inclusion. Five of these papers met all predefined eligibility criteria. Introducing methods to enhance exercise adherence has caused a significant increase in exercise adherence for less than 6 or 12 months. There were no significant differences in adherence for more than 12 months with different methods. The results indicate that booster-sessions (89.69\%) and telephone-linked communication (86\%) had higher percentages for exercise adherence. Secondary outcomes such as pain, stiffness and function show positive outcomes with increasing exercise adherence. However, there were no significant differences on these secondary outcomes.
\end{abstract}

Conclusion: The booster sessions and telephone-linked communication appear to enhance exercise adherence for more than 12 months among patients with $\mathrm{OA}$. However, the number of high-quality studies is inadequate to confirm

*Correspondence: cinthuja@med.cmb.ac.lk

${ }^{1}$ Department of Allied Health Sciences, Faculty of Medicine, University

of Colombo, Colombo 00800, Sri Lanka

Full list of author information is available at the end of the article

(c) The Author(s) 2022. Open Access This article is licensed under a Creative Commons Attribution 4.0 International License, which permits use, sharing, adaptation, distribution and reproduction in any medium or format, as long as you give appropriate credit to the original author(s) and the source, provide a link to the Creative Commons licence, and indicate if changes were made. The images or other third party material in this article are included in the article's Creative Commons licence, unless indicated otherwise in a credit line to the material. If material is not included in the article's Creative Commons licence and your intended use is not permitted by statutory regulation or exceeds the permitted use, you will need to obtain permission directly from the copyright holder. To view a copy of this licence, visit http://creativecommons.org/licenses/by/4.0/. The Creative Commons Public Domain Dedication waiver (http://creativeco mmons.org/publicdomain/zero/1.0/) applies to the data made available in this article, unless otherwise stated in a credit line to the data. 
our findings. Therefore, more studies with higher methodological quality are needed to determine the best strategies to enhance long-term exercise adherence among people with OA.

Keywords: Osteoarthritis, Long term, Exercise adherence

\section{Background}

Osteoarthritis (OA) is defined as a 'clinical syndrome of joint pain accompanied by varying degrees of functional limitation and reduced quality of life' [1]. This is characterized by 'pathological loss of cartilage, remodelling of adjacent bone and associated inflammation' [1]. OA can develop as primary osteoarthritis and secondary osteoarthritis where predisposing conditions exist. This is one of the important causes of disability and pain [2]. OA has an impact on individuals, society and the health care system. In terms of individuals, it affects a person's quality of life by causing pain and reduction in function. OA can affect joints in peripheral either single or multiple joints. Small hand joints, hips and knees are commonly affected joints [1].

$\mathrm{OA}$ is managed through non-pharmacological and pharmacological methods. Guidelines have been developed to manage OA. Many guidelines have been published for the management of OA by institutions such as NICE (National Institute for Clinical Excellence), OARSI (Osteoarthritis Research Society International), and EULAR (European League Against Rheumatism). According to the NICE guidelines, education, advice, information access, exercises for strengthening, aerobic fitness and weight loss should be considered as core treatments [1]. Paracetamol and topical NSAIDs should be considered when further treatment is needed [1]. Other options include self-management techniques (local heat and cold, assistive devices), pharmaceutical options and surgery (joint arthroplasty) and non-pharmaceutical treatments (support and braces, shockabsorbing shoes or insoles, TENS and manual therapy), whose efficacy is less well proven, offers less symptom relief and may carry risks for patients [1].

The World Health Organization (WHO) defines 'the extent to which a person's behaviour corresponds with agreed recommendations from a healthcare provider' as adherence [3]. Adherence to guidelines by health care providers and patients is an important factor for the effective management of OA. In patients with osteoarthritis, a low level of adherence to physiotherapy exercise affects the effectiveness and outcomes of prescribed exercise [4-6]. It is important to study effective methods to enhance adherence to core management methods, especially long-term adherence to exercises by OA patient groups.
One of the significant challenges in chronic and longterm conditions is adherence to the exercises or management guidelines. Supervised exercise sessions, refresher sessions, audio or videotapes of exercise programmes, self-management programme and cognitive adherence measures are methods that have been shown to improve exercise adherence [7]. The study findings concluded that supervised and individual exercise therapy and self-management techniques increase exercise adherence among adults with chronic musculoskeletal pain [7]. A Cochrane review suggested self-management techniques, supervised and individualized exercise programmes, activity monitoring, feedback systems, written instructions and behavioural exercise programmes, booster sessions, behavioural graded exercises and peer-delivered programmes have shown adherence potential [3]. A study conducted in Australia to identify the barriers and enablers for the management of osteoarthritis stated that ongoing episodic nature, the availability of visual evidence of joint surface damage on X-ray images and prescribing exercise when it is perceived as a reason for joint surface damage are challenges in the management of OA. This study suggested that clinician adherence to guidelines can be enhanced by decision support and reminder systems, interactive educational strategies and educational outreach and clinical practice audits that provide feedback to clinicians [8].

$\mathrm{OA}$ is a chronic condition. It affects people's mobility and the quality of life. Joint pain, stiffness, decreased range of joint movement, muscle weakness of quadriceps and alterations in proprioception are clinical manifestations of OA [9]. Studies have shown significant benefits of exercise in patients with osteoarthritis, especially exercises focusing on strength, flexibility and aerobic capacity [10]. International guidelines from institutions such as NICE, EULAR and OARSI recommend core exercises and effective management $[1,10]$, which are key elements of any treatment program for OA [11]. Regular exercise reduces physical impairments and improvements in mobility and mental health $[10,12]$. This improves everyday physical function. There are non-pharmaceutical treatments such as support and braces, shock-absorbing shoes or insoles, TENS, and manual therapy; however, these are less well proven in terms of efficacy, offer less symptom relief and carry risks for patients according to NICE guidelines [1]. Long-term clinical success is based on long term adherence to a recommended exercise 
programme $[6,13]$. Life-long exercise is considered crucial for maximizing the well-being and function of adults with knee OA [14]. However, whether patients adhere to these exercises in the long term is not clear [5]. Therefore, it is essential to study adherence to core management methods (especially adherence to physiotherapy exercise by patients with osteoarthritis), identify methods to enhance long-term adherence to these guidelines for more than 12 months, and follow up to monitor or evaluate the level of adherence to guidelines and investigate the effectiveness of the intervention methods to enhance the exercise adherence among patients with osteoarthritis. Many reviews have been conducted to assess exercise adherence for $\leq 12$ months $[7,15]$. This study aimed to determine the methods to enhance long term (i.e. more than 12 months) exercise adherence among patients with lower limb osteoarthritis and to identify the most effective methods to enhance exercise adherence among patients with osteoarthritis.

\section{Methodology}

A systematic review was performed to identify effective methods to enhance long-term ( $>12$ months) exercise adherence among patients with lower limb osteoarthritis. This systematic review was conducted according to the Preferred Reporting Items of Systematic Reviews and Meta-Analyses (PRISMA) guidelines [16]. This protocol was registered (the protocol was not peer reviewed and registration during the 2020 pandemic was automatically published exactly as submitted) in Prospero (Prospero ID: CRD42020205653).

\section{Search strategy}

A literature search was performed using electronic databases on 27 August 2020 (PubMed, Pedro, EMBASE and Web of Science). Databases were searched for randomized controlled trials, cohort studies, case-control studies, and cross-sectional studies published in English from 2000 to 2020 . The search strategy included only terms relating to or describing the interventional methods. The keywords used for the search were as follows: osteoarthritis, arthritis, hip, lower limb, knee, extended period, long period, compliance (Table 1), adherence (Table 1), follow-up, engagement (Table 1), strengthening, exercise, physical therapy, rehabilitation therapy, resistance training, muscle strengthening and aerobic exercise. Full search strategy and individual results can be found in electronics supplement. The searches were rerun just before the final analyses and further studies were retrieved for inclusion. The date of data extraction was 4 September 2020. Cited articles among included studies were also checked.
Table 1 Operational definition

\begin{tabular}{ll}
\hline Term & Operational definition \\
\hline Compliance & $\begin{array}{l}\text { the fact of obeying a particular law or rule, } \\
\text { or of acting according to an agreement } \\
\text { the fact of being involved with something: } \\
\text { Engagement: }\end{array}$ \\
the act of doing something according to a \\
particular rule, standard, agreement
\end{tabular}

\section{Selection criteria}

Full papers were included in the final analysis. Randomized controlled trials (RCTs), cohort studies, casecontrol studies, cross-sectional studies published in English and peer-reviewed journals were eligible for inclusion.

Studies conducted among the population with lower limb knee or hip joint osteoarthritis that recruited both males and females aged above 18 years, carried out physiotherapy exercise as a primary intervention and followed up for more than 12 months were eligible for inclusion. Studies including lower limb muscle pathology or systemic or metabolic or neurological disease were excluded.

Therapeutic exercise is the systematic, planned performance of bodily movements, postures or physical activities intended to provide a patient/client with the means to remediate or prevent impairments; improve, restore or enhance physical function; prevent or reduce healthrelated risk factors; and optimise overall health status, fitness or sense of well-being [17]. Physical therapists select, prescribe and implement exercise activities when the examination findings, diagnosis and prognosis indicate the use of therapeutic exercise $[18,19]$. Therapeutic exercises for osteoarthritis include strengthening, stretching and cardiovascular exercises [20,21].

\section{Outcome}

Articles were included if the intervention(s) aimed to improve adherence, compliance or engagement with exercise, compared with either no adherence or engagement intervention. A paper with a comparator group that was also undertaking the exercise programme and where a no-intervention control group occurred for a long duration but there were at least two active intervention groups to offer a comparison was also included.

\section{Data extraction}

Two independent researchers retrieved titles and abstracts of studies using the search strategy and those from additional sources, i.e. cited articles among included studies, were screened independently by two reviewers (CP \& NK). A pilot test was done on the data extraction sheet and based on that a placeholder section 
for final results of the studies and methodology quality assessment results were added to the final data extraction form. The full text of these potentially eligible studies was retrieved and independently assessed for eligibility. Disagreements were resolved through discussion with a third reviewer (GS).

A predetermined extraction form was used to extract data from the included studies to assess study quality and evidence synthesis. Study setting, study population, participant demographics, details of the intervention, methods used to enhance exercise adherence and study methodology were extracted independently by two review authors. Discrepancies were identified and discussed between the two researchers (CP \& NK) to resolve any differences of opinion, and a third reviewer's opinion was taken when it was not possible to reach a consensus. Relevant authors were requested to provide missing data; one such attempt by investigators was made via email.

\section{Quality and risk of Bias assessment}

Two reviewers independently conducted methodology quality assessment using the Pedro scale, and the Risk of Bias Assessment tool for randomised control trials, RoB2 [22], was used to assess the risk of bias (Table 2). The PEDro scale is composed of 11 items, of which the first item is only applicable for specification of eligibility criteria, and it was not considered as part of calculating the overall PEDro score. Each item was given one point, and the total score could be between 0 to 10 points. Studies that scored $\geq 4$ points were considered 'high' quality and studies that scored $<4$ points were considered to be of 'low' methodological quality [23]. Studies heterogeneity checked for potential meta-analysis. RoB2 focuses on different aspects of trail design, conduct and reporting. Judgment about risk of bias arising from each domain is generated by an algorithm, based on the questions. Domains include bias arising from the randomization process, bias arising from the timing of identification or recruitment of participants, bias due to deviation from the intended interventions (effect of assignment to intervention), bias due to deviations from the intended interventions (effect of adhering to intervention), bias due to missing outcome data, bias due to measurement of the outcome and bias in selection of the report result. Based on the assessment, a domain can be 'low' or 'high' risk of bias or it can be expressed as 'some concerns' [24].

Cohen's Kappa analysis was performed to identify the agreement between raters on the methodology quality of the included studies. The percentage of agreement on methodological quality was noted as $80 \%$. Moderate interrater reliability with $\mathrm{k}=0.72, \mathrm{SE}=0.21$ [25], was

Table 2 The table shows risk of bias assessment using Risk of Bias Assessment tool for systematic reviews tool

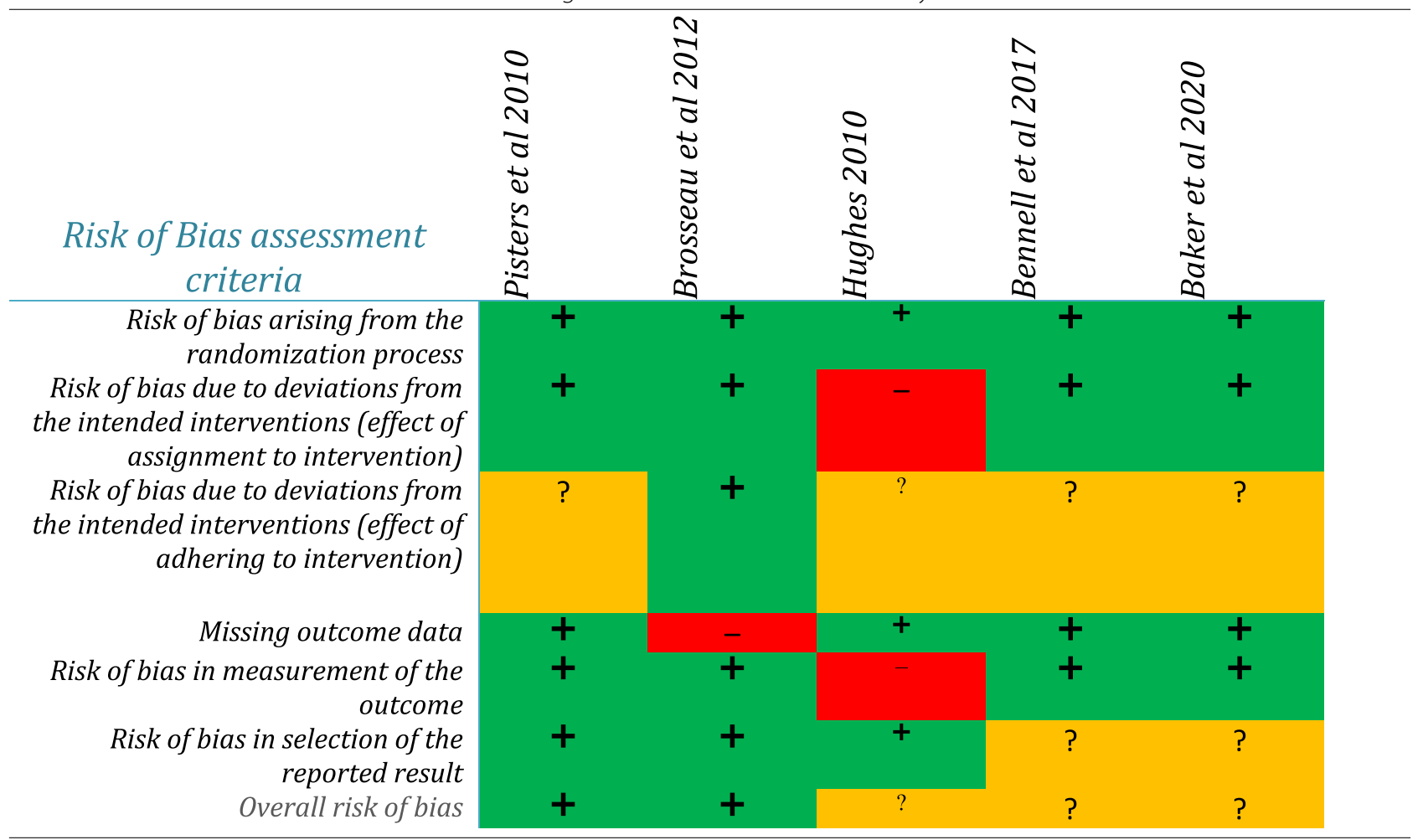

Low risk of bias (green), high risk of bias (red), unclear risk of bias (amber) 
analysed using SPSS (statistics version 26.0.0; SPSS Inc., Chicago, IL).

\section{Results}

\section{Selection of studies}

The primary search strategy identified 5839 potentially relevant articles of which 5157 remained after discarding duplicates (Fig. 1). The date of data extraction was 4 September 2020. After screening based on title and abstract, 40 papers were potentially eligible for inclusion. The number of additional articles from external searches was zero. Five of these papers met all the predefined eligibility criteria.

Studies were excluded due to many reasons, the most common reason being the follow-up duration. A follow up of more than 12 months was not conducted for most of the trials. Studies were also excluded when physiotherapy exercise was not the intervention for patients with osteoarthritis or there was no intervention to improve exercise adherence.

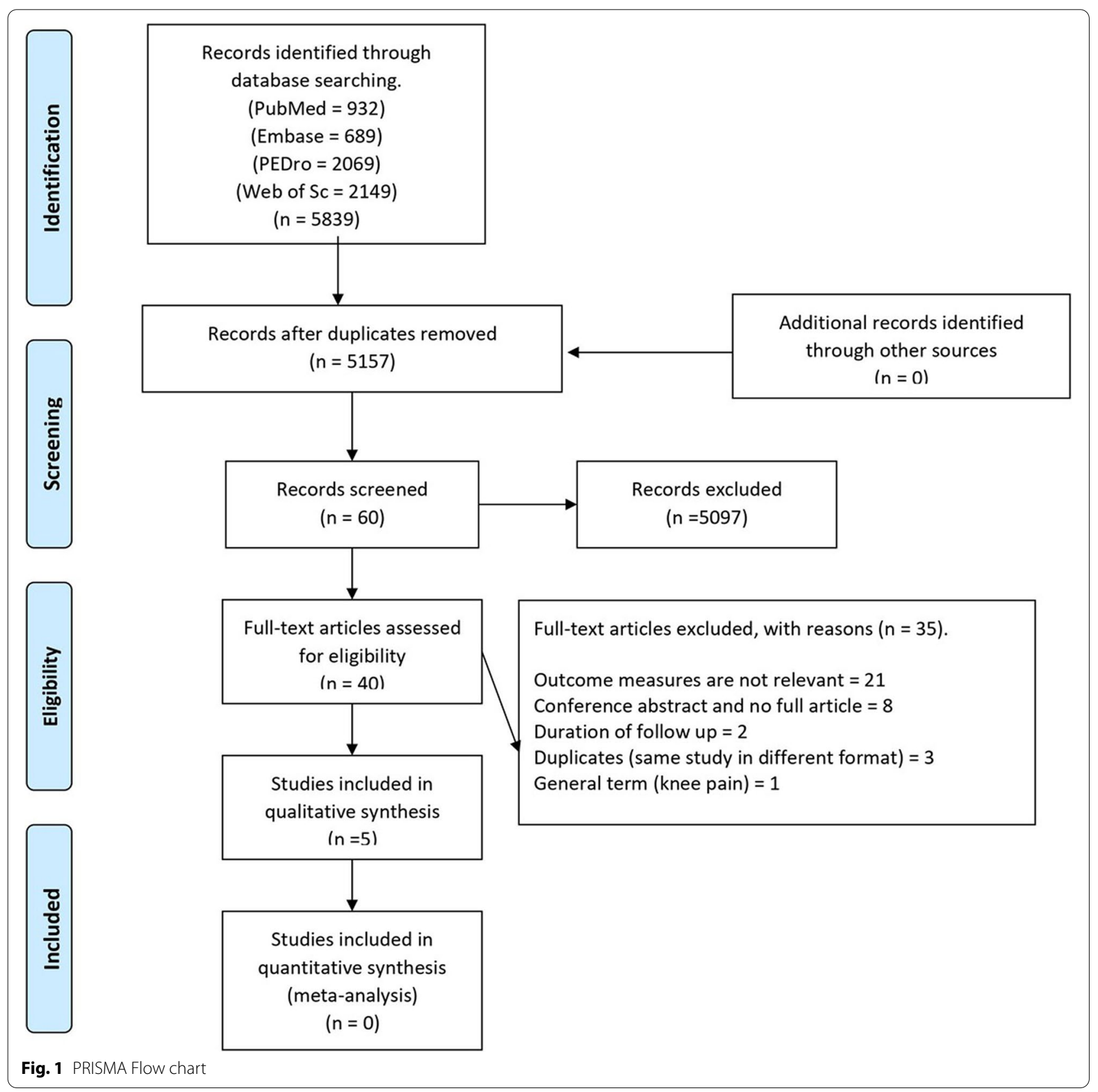




\section{Characteristics of included studies}

In total, 1113 patients with lower limb osteoarthritis were included in the five studies that were analysed. All studies used an RCT design and included patients who were currently diagnosed with knee osteoarthritis, hip osteoarthritis or both. The median sample size was 223 participants (range $=104-419$ ). The mean age was 65.37 $( \pm 3.42)$ years. The mean length of the follow-up was 26.6 months (range $=18-55$ months). Of the five studies, four reported using the intention-to-treat (ITT) method for missing data, and one study did not confirm the type of analysis used. In the selected five studies, booster sessions, the behavioural approach, telephone sessions, motivation calls and telephone reinforcement were used to enhance exercise adherence.

In the five studies, mean duration of exercise interventions was 19.6 weeks (range $=6-48$ weeks). Interventions include individually-tailored exercises with complete protocols including written materials such as education messages, activity diaries, performance charts [6], walking sessions [26], $60 \mathrm{~min}$ of stretching/flexibility, lowimpact aerobics, strengthening and balance exercises followed by $30 \mathrm{~min}$ of manual-based, group problemsolving/health education for managing OA with PA [27], 30-min consultations with a physiotherapist over six months for education, home exercise and physical activity advice [28] and six-week group exercise classes [29].

In the five studies, the behavioural approach at a community-based walking club [26], telephone sessions with a health coach [28], TLC motivational calls [29], telephone reinforcement [27] and booster sessions [6] were the strategies used to enhance exercise adherence among participants in the experimental groups. The adherence rate for the five-booster session group was $89.69,59.4 \%$ for the behavioural approach group, 39\% for 6-12 telephone sessions with a health coach and $86 \%$ for the telephone-linked communication group. Individual scores were not available for the telephone reinforcement group.

In addition to exercise adherence, physical activity level, lower extremity pain, stiffness, function, sit-stand, a six-minute distance walk, anxiety, or depression, the Western Ontario and McMaster Universities Arthritis Index (WOMAC) pain, WOMAC physical activity, function subscale, functional performance assessment, quadriceps and hamstring strength were other outcomes measured in these studies.

\section{Assessment of methodological quality and quantitative analysis}

The five included studies were scored using the PEDro scale. Methodological quality ranged from 3 to 8 as rated on the PEDro scale with a median score of 6.8. The methodology quality assessment is summarized in Table 3 . All studies were rated as high qualitative studies with a score of $\geq 4$ [23], of which 2 scored $8 / 10$ points and 2 scored $7 / 10$ points. Quantitative analysis by pooling outcome data (meta-analysis) or a best-evidence synthesis was inappropriate. This is due to the incomparability of outcome data caused by the heterogeneity of techniques to enhance exercise adherence.

Table 4 summarizes the characteristics of the studies included in the analysis. The domains commonly lacking in the included studies were as follows: the therapists who administered the treatment were blinded, measures of key outcomes were obtained from more than $85 \%$ of subjects and subjects were blinded.

Table 3 Table shows methodological quality assessment of included studies assessed using Pedro scale

\begin{tabular}{|c|c|c|c|c|c|}
\hline Methodology quality assessment & $\begin{array}{l}\text { Pisters et al } \\
2010 \text { [6] }\end{array}$ & $\begin{array}{l}\text { Brosseau et al } \\
2012[23]\end{array}$ & $\begin{array}{l}\text { Hughes } \\
\text { et al } 2010 \\
{[27]}\end{array}$ & $\begin{array}{l}\text { Bennell et al } \\
2017 \text { [25] }\end{array}$ & $\begin{array}{l}\text { Baker } \\
\text { et al } 2020 \\
{[26]}\end{array}$ \\
\hline Eligibility criteria were specified (Not used in score generation) & Y & Y & Y & Y & Y \\
\hline Subjects were randomly allocated to groups & Y & Y & Y & Y & Y \\
\hline Allocation was concealed & Y & Y & NG & Y & Y \\
\hline Groups were similar at baseline & Y & Y & Y & Y & NG \\
\hline Subjects were blinded & Y & $\mathrm{N}$ & $\mathrm{N}$ & Y & NG \\
\hline Therapist who administered the treatment were blinded & $\mathrm{N}$ & $\mathrm{N}$ & $\mathrm{N}$ & $\mathrm{N}$ & $\mathrm{N}$ \\
\hline Assessors were blinded & Y & Y & $\mathrm{N}$ & Y & Y \\
\hline Measures of key outcomes were obtained from more than $85 \%$ of subjects & N & N & $\mathrm{N}$ & N & Y \\
\hline Data were analysed by intention to treat & Y & Y & NG & Y & Y \\
\hline Statistical comparisons between groups were conducted & Y & Y & Y & Y & Y \\
\hline Points measures and measures of variability were provided & Y & Y & NG & Y & Y \\
\hline Total score & 8 & 7 & 3 & 8 & 7 \\
\hline
\end{tabular}

$Y$ Yes, N No, NG Not given 


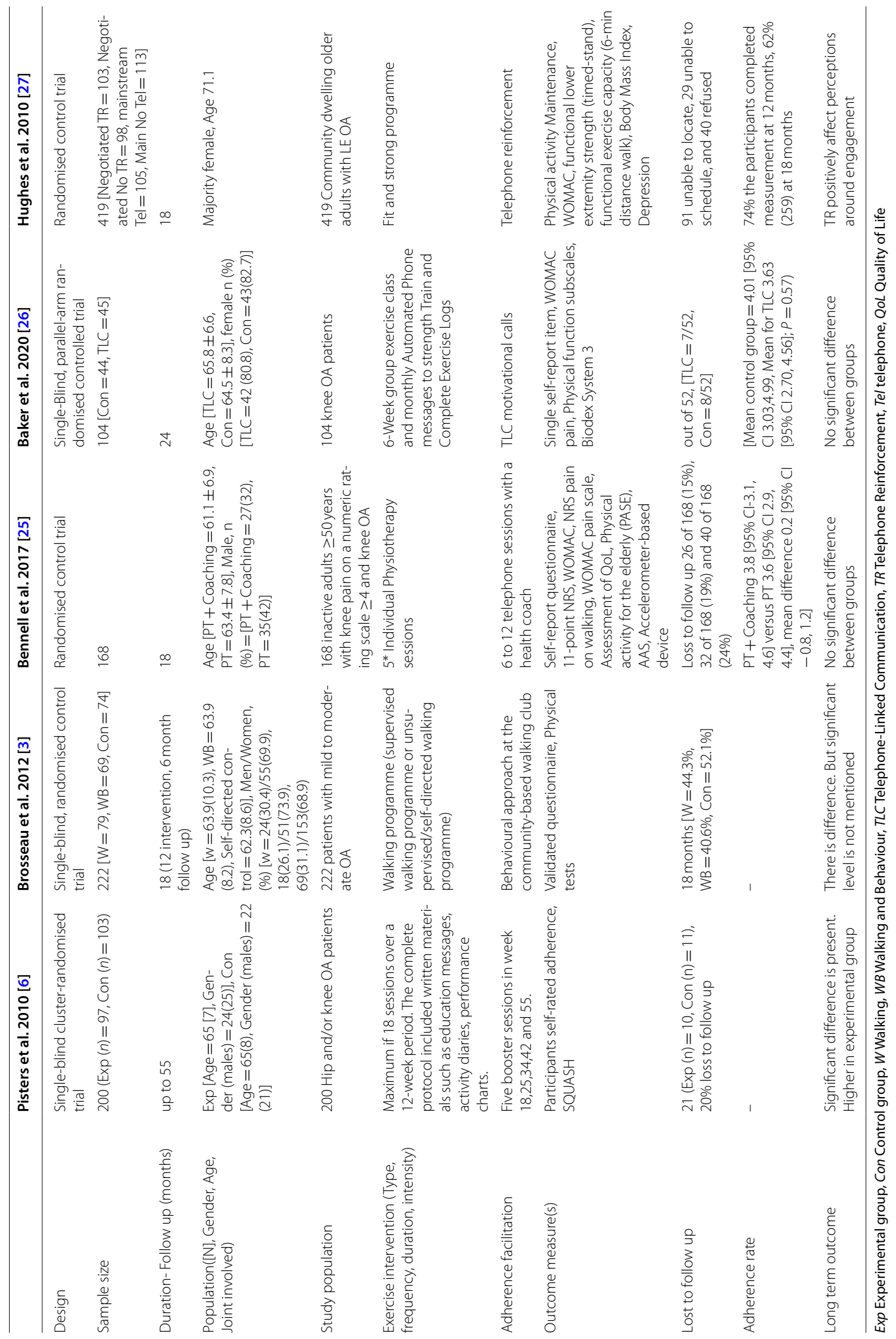




\section{Measurement instruments and outcome measures of adherence}

Adherence to exercise intervention was measured using a questionnaire [6], the number of attended walking sessions divided by the number of prescribed sessions recorded by the exercise therapist and participants' completed logbooks [26], self-report questionnaires completed at home at baseline, 6, 12 and 18 months [28], single self-report item interviewer-administered at 12 and 24 months and administered by phone at 18 months [29] and self-administrated questionnaires [30]. Out of these five studies, four have reported adherence as one of the primary outcomes $[6,26,29,30]$.

\section{Discussion}

This review summarizes different methods to enhance exercise adherence among people with knee osteoarthritis for more than 12 months. Different factors were reported for loss of follow-up with the exercise programme. This section will focus on the main findings related to the study.

\section{Summary of main findings}

Five studies were eligible according to the inclusion criteria. Strategies used to enhance exercise adherence vary among trials. Booster sessions, behavioural approaches, telephone sessions, telephone linked communication, motivational calls and telephone reinforcement were used as strategies in these studies.

The long-term exercise adherence rate of the following methods varied between studies. According to the results, booster sessions and telephone-linked communication had higher percentages for exercise adherence. The experimental group who received booster sessions had a maximum of 18 sessions (individually-tailored exercises, education message, activity diaries and performance chart) over a 12-week period, followed by five booster sessions in weeks $18,25,34,42$, and 55 [6]; the experimental group who received telephone linked communication received five 30 -min consultations with a physiotherapist over 6 months for education, home exercise, physical activity advice and 6-12 telephone coaching sessions by a clinician trained in behavioural change support for exercise and physical activity [28]. Previous studies suggested behavioural strategies and educational approaches will improve exercise adherence [15, 31]. Exercise adherence is influenced by different factors. These can be divided into intrinsic/personal factors or extrinsic factors. Health care and well-informed motivated health care providers, lifestyle-related issues and the extent of ongoing social or therapist contacts, knowledge about their exercise regimen and its potential effectiveness and degree of self-motivation are some of the factors related to exercise adherence [15]. During booster sessions and telephone linked communication, some of these factors are addressed, mainly behavioural change and knowledge and ongoing therapist contacts, which increase exercise adherence. Therefore, future studies should investigate the effectiveness of these two strategies in enhancing exercise adherence.

According to the systematic review results, introducing methods to enhance exercise adherence has only a shortterm impact. There are no significant differences in longterm adherence with different methods. Further, most of the secondary outcomes show positive outcomes with increasing exercise adherence. However, there are no significant differences in outcomes between the experimental and control groups. The non-significant difference might be due to a lack of adherence to the exercise guidelines. In the future, it is recommended that an individual patient should be assessed and the potential reasons for lack of adherence; for example, lack of motivation, socioeconomic situation, family and work commitments, should be assessed when formulating exercise adherence strategies for an individual. This might potentially enhance their exercise adherence.

Outcome measures were used to measure exercise adherence in the selected studies. Most of the studies used a self-rated adherence rate. In previous reviews similar methods were used to measure adherence rate [15]. Self-rated adherence rate might have a bias as it depends on a participant's response. There is a possibility for overestimation of exercise through recall or social desirability bias [15]. Therefore, a standard method, such as attendance records and validated wearable activity trackers, should be used in future studies to report exercise adherence rates [23].

Commonly reported reasons for loss of follow up were co-morbidity, selected surgical intervention option, family circumstances, decline to participate, lack of motivation, pain increase, loss of contact, withdrawal from the study due to personal reasons, other illness, family illness, lack of time, change in location and death. It is reported that the barriers to continuing with the interventions were weather conditions and health problems of participants' partners. A study was conducted to determine the predictors of adherence to exercise interventions during and after cancer treatment. According to the results, prominent predictors of exercise adherence were the location of the rehabilitation centre and motivation for exercise [23]. These factors are similar to people with osteoarthritis. Studies among people with OA indicated that pain, mental health, body mass index, important problems associated with disabling knee osteoarthritis, the extent to which patients are compensated for their disability, prior exercise behaviours, exercise beliefs and 
knowledge and lack of time are associated with exercise adherence [14, 32-34]. Therefore, it is important to consider these barriers and reasons when identifying interventions to enhance exercise adherence among people with long-term conditions.

This review finding is consistent with previous studies. The existing literature suggests that there is a significant difference in short-term exercise adherence but then patients fail to maintain adherence in long term [15, 33]. It is mentioned that it is difficult to determine which component of the intervention was truly having a positive effect on adherence [34]. These findings are similar to this study's findings. Further, the literature suggests that there are different determinants [14] for exercise adherence that include both intrinsic and extrinsic factors, and there is no single strategy that can be effective in promoting adherence among people with arthritis [14]. The studies included in the current review used types of intervention that addressed different intrinsic and extrinsic factors. All included studies addressed either one of the intrinsic/extrinsic factors or both factors. Lack of adherence to exercise is the main reason for decline in benefits over time and their disappearance in the long term. Therefore, there is a limited difference in longterm follow up results of these studies. In this review, it appears that mainly behavioural change, awareness about their condition and ongoing therapist contacts have an influence on increasing exercise adherence. Moreover, it should be noted that behavioural patterns are more likely to differ for a larger population. Therefore, it is important to consider that fact when formulating an intervention strategy.

\section{Strengths}

One of the strengths of this systematic review is that four studies were of 'high' methodological quality. This systematic review used the PRISMA guidelines for reporting systematic review and the PEDro scale.

\section{Limitations}

Several limitations should be considered during this study's interpretation and be addressed in future research. First, only five studies that assessed exercise adherence for more than 12 months were included in this systematic review. Different exercise interventions and exercise adherence strategies were used in these five studies with variations in duration and intensity. The heterogeneity is noted in these studies. Therefore, quantitative analysis by pooling outcome data (meta-analysis) or a best evidence synthesis was inappropriate. The interrater reliability is noted as 0.72 because of the small number of studies included in this study.
In the existing literature, only a limited number of studies focused on exercise adherence for more than 12 months. Also, self-reported exercise adherence was used to assess the adherence rate. Exercises should be followed as one of their routine activities among people with chronic conditions such as osteoarthritis. Many studies reported exercise adherence for a period of less than 12 months or as long as patients actively participate in clinical visits. However, follow up should continue for a longer duration to ensure that patients achieve the benefits of the prescribed exercise programme. These limitations should be addressed in future studies.

The study only included articles published in English. An extensive database review was conducted using a broad search strategy. However, there is a possibility for publication bias and selective reporting biases.

\section{Future research}

Only five studies were eligible according to the criteria. Therefore, in the future, more studies should be conducted to identify the best strategies to maintain exercise adherence for more than 12 months among people with osteoarthritis. In addition, attendance records and validated wearable activity trackers should be used to calculate exercise adherence among participants.

According to the present study, booster sessions and telephone linked communication showed a higher adherence rate. It appears that mainly behavioural change strategies and awareness about their condition and ongoing therapist contacts have an influence on increasing exercise adherence. It is suggested that future studies investigate further these two techniques for long term exercise adherence among OA patients or in any chronic conditions that require continuous exercise adherence.

In this review, articles published in English language were included. In the future, publications in all languages should be considered.

\section{Clinical implications}

This review findings suggest that behavioural changes strategies, patient awareness about their condition and continuous therapist contacts have a positive influence on long-term exercise adherence. Therefore, clinicians should incorporate behavioural changes strategies in their management in addition to exercise prescription. Clinician should educate their patients about the nature of $\mathrm{OA}$ and the importance of long-term exercise adherence to achieve good outcomes from exercise programme.

Success of rehabilitation protocol implies on long term adherence of exercise programme, and this review suggests that having a periodical contact with person with OA would provide massive clinical benefits. 


\section{Conclusion}

According to the results, booster sessions and telephonelinked communication had higher percentages for exercise adherence. Results indicated that utilizing different strategies does not have a significant influence on the exercise adherence a period of more than 12 months and a non-significant difference in the primary and secondary outcomes. However, the number of high-quality studies is inadequate to confirm our findings. Therefore, high quality future studies are needed to determine the best strategy to enhance long-term exercise adherence among patients with osteoarthritis.

\section{Abbreviations \\ OA: Osteoarthritis; NICE: National Institute for Clinical Excellence; OARSI: Osteo- arthritis Research Society International; EULAR: European League Against Rheumatism; WHO: World Health Organization; PRISMA: Preferred Reporting Items of Systematic Reviews and Meta-Analyses; ITT: Intention-To-Treat; TLC: Telephone linked Communication; WOMAC: Western Ontario and McMaster Universities Arthritis Index; Exp: Experimental group; Con: Control group; W: Walking; WB: Walking and Behaviour; TR: Telephone reinforcement; Tel: Telephone; QoL: Quality of Life.}

\section{Supplementary Information}

The online version contains supplementary material available at https://doi. org/10.1186/s12891-022-05050-0.

\section{Additional file 1.}

\section{Acknowledgements}

Not applicable.

\section{Authors' contributions}

Conception and design of the study: CP, GS, Screening of the articles: CP, NK and GS; Data extraction: CP and NK; Methodological quality ratings and data analysis: CP and NK; First drafting of the manuscript: CP and NK; Reviewing article: GS, CP and NK; Editing: CP and NK. All authors approved the version to be published.

\section{Funding}

No funding was received for this study.

\section{Availability of data and materials}

Supplementary file is attached with search strategies. The datasets used and/ or analyzed during the current study are available from the corresponding author [CP] on reasonable request.

\section{Declarations}

\section{Ethics approval and consent to participate}

It was not taken as in this study no participants were recruited. Consent was not taken as in this study no participants were recruited.

\section{Consent for publication \\ Not applicable.}

\section{Competing interests}

Authors declare no competing interests.

\section{Author details}

${ }^{1}$ Department of Allied Health Sciences, Faculty of Medicine, University of Colombo, Colombo 00800, Sri Lanka. ${ }^{2}$ Physio Medicare, Private
Physiotherapy clinic, Dehiwala 10350, Sri Lanka. ${ }^{3}$ School of Engineering and Material Science, Queen Mary University of London, Bancroft Road, Mile End, London E1 4NS, UK.

Received: 22 September 2021 Accepted: 19 January 2022 Published online: 14 February 2022

\section{References}

1. N I C E. National Institute for health and care excellence. Osteoarthritis: Care and management, Clinical Guideline. 2014. https://www.nice.org.uk/ guidance/cg177.

2. Healey EL, Afolabi EK, Lewis M, Edwards JJ, Jordan KP, Finney A, et al. Uptake of the NICE osteoarthritis guidelines in primary care: a survey of older adults with joint pain. BMC Musculoskelet Disord. 2018;19(1):295.

3. Room J, Hannink E, Dawes H, Barker K. What interventions are used to improve exercise adherence in older people and what behavioural techniques are they based on? A systematic review. BMJ Open. 2017;7(12):e019221.

4. Argent R, Daly A, Caulfield B. Patient involvement with home-based exercise programs: can connected health interventions influence adherence? JMIR MHealth UHealth. 2018;6(3):e47.

5. Nicolson PJA, Hinman RS, Kasza J, Bennell KL. Trajectories of adherence to home-based exercise programs among people with knee osteoarthritis. Osteoarthr Cartil. 2018;26(4):513-21.

6. Pisters MF, Veenhof C, de Bakker DH, Schellevis FG, Dekker J. Behavioural graded activity results in better exercise adherence and more physical activity than usual care in people with osteoarthritis: a cluster-randomised trial. J Physiother. 2010;56(1):41-7.

7. Jordan JL, Holden MA, Mason EE, Foster NE. Interventions to improve adherence to exercise for chronic musculoskeletal pain in adults. Cochrane Database Syst Rev. 2010;1:CD005956.

8. Egerton T, Diamond LE, Buchbinder R, Bennell KL, Slade SC. A systematic review and evidence synthesis of qualitative studies to identify primary care clinicians' barriers and enablers to the management of osteoarthritis. Osteoarthr Cartil. 2017;25(5):625-38.

9. Carvalho NA, Bittar ST, Pinto FR, Ferreira M, Sitta RR. Manual for guided home exercises for osteoarthritis of the knee. Clinics (Sao Paulo). 2010;65(8):775-80.

10. Uthman OA, van der Windt DA, Jordan JL, Dziedzic KS, Healey EL, Peat GM, et al. Exercise for lower limb osteoarthritis: systematic review incorporating trial sequential analysis and network meta-analysis. BMJ. 2013;347:f5555

11. Luan X, Tian X, Zhang H, Huang R, Li N, Chen P, et al. Exercise as a prescription for patients with various diseases. J Sport Health Sci. 2019;8(5):422-41.

12. Alghamdi MAA, Olney S, Costigan P. Exercise treatment for osteoarthritis disability. Ann Saudi Med. 2004;24(5):326-31.

13. Tuakli-Wosornu YA, Selzer F, Losina E, Katz JN. Predictors of exercise adherence in patients with meniscal tear and osteoarthritis. Arch Phys Med Rehabil. 2016;97(11):1945-52.

14. Marks R. Knee osteoarthritis and exercise adherence: A review. Curr Aging Sci. 2012;5(1):72-83.

15. Ezzat AM, MacPherson K, Leese J, Li LC. The effects of interventions to increase exercise adherence in people with arthritis: a systematic review: exercise adherence interventions in arthritis. Musculoskeletal Care. 2015;13(1):1-18.

16. Liberati A, Altman DG, Tetzlaff J, Mulrow C, Gøtzsche PC, loannidis JPA, et al. The PRISMA statement for reporting systematic reviews and metaanalyses of studies that evaluate healthcare interventions: explanation and elaboration. BMJ. 2009:339:b2700.

17. Allen CL, Carolyn K. Therapeutic Exercise- Foundation techniques. 6th ed. Philadelphia: F. A. Davis Company;

18. Osteoarthritis $(\mathrm{OA})$ of the knee [Internet]. Versusarthritis.org. [cited 2021 Oct 24]. Available from: https://www.versusarthritis.org/about-arthritis/ conditions/osteoarthritis-of-the-knee/

19. Quality statement 4: Exercise | Osteoarthritis | Quality standards | NICE. [cited 2021 Oct 24]; Available from: https://www.nice.org.uk/guidance/ qs87/chapter/quality-statement-4-exercis 
20. Büttner F, Winters M, Delahunt E, Elbers R, Lura CB, Khan KM, et al. Identifying the 'incredible'! Part 2: spot the difference - a rigorous risk of bias assessment can alter the main findings of a systematic review. Br J Sports Med. 2020;54(13):801-8.

21. Ormel HL, van der Schoot GGF, Sluiter WJ, Jalving M, Gietema JA, Walenkamp AME. Predictors of adherence to exercise interventions during and after cancer treatment: A systematic review. Psychooncology. 2018;27(3):713-24.

22. McHugh ML. Interrater reliability: the kappa statistic. Biochem Med (Zagreb). 2012;22(3):276-82.

23. Brosseau L, Wells GA, Kenny GP, Reid R, Maetzel A, Tugwell P, et al. The implementation of a community-based aerobic walking program for mild to moderate knee osteoarthritis (OA): a knowledge translation (KT) randomized controlled trial (RCT): part I: the uptake of the Ottawa panel clinical practice guidelines (CPGs). BMC Public Health. 2012;12(1):871.

24. Desai PM, Hughes SL, Peters KE, Mermelstein RJ. Impact of telephone reinforcement and negotiated contracts on behavioral predictors of exercise maintenance in older adults with osteoarthritis. Am J Health Behav. 2014;38(3):465-77.

25. Bennell KL, Campbell PK, Egerton T, Metcalf B, Kasza J, Forbes A, et al. Telephone coaching to enhance a home-based physical activity program for knee osteoarthritis: A randomized clinical trial. Arthritis Care Res (Hoboken). 2017;69(1):84-94.

26. Baker K, LaValley MP, Brown C, Felson DT, Ledingham A, Keysor JJ. Efficacy of computer-based telephone counseling on long-term adherence to strength training in elderly patients with knee osteoarthritis: A randomized trial. Arthritis Care Res (Hoboken). 2020;72(7):982-90.

27. Hughes SL, Seymour RB, Campbell RT, Desai P, Huber G, Chang HJ. Fit and Strong!: bolstering maintenance of physical activity among older adults with lower-extremity osteoarthritis. Am J Health Behav. 2010;34(6):75063. https://doi.org/10.5993/ajhb.34.6.10.

28. Roter DL, Hall JA, Merisca R, Nordstrom B, Cretin D, Svarstad B. Effectiveness of interventions to improve patient compliance: a meta-analysis. Med Care. 1998;36(8):1138-61.

29. Thomas KS, Muir KR, Doherty M, Jones AC, O'Reilly SC, Bassey EJ. Home based exercise programme for knee pain and knee osteoarthritis: randomised controlled trial. BMJ. 2002;325(7367):752.

30. Kolt GS, McEvoy JF. Adherence to rehabilitation in patients with low back pain. Man Ther. 2003;8(2):110-6.

31. Hendry M, Williams NH, Markland D, Wilkinson C, Maddison P. Why should we exercise when our knees hurt? A qualitative study of primary care patients with osteoarthritis of the knee. Fam Pract. 2006;23(5):558-67.

32. Rejeski WJ, Brawley LR, Ettinger W, Morgan T, Thompson C. Compliance to exercise therapy in older participants with knee osteoarthritis: implications for treating disability. Med Sci Sports Exerc. 1997;29(8):977-85.

33. Berg MH, Hk R, Aj P, Cessie S, Giesen FJ, Fc B. Using internet technology to deliver a home-based physical activity intervention for patients with rheumatoid arthritis: A rondomised controlled trial. Vliet Vlieland T s I: Arthritis and Rheumatism. 2006;55:935-45.

34. Talbot LA, Gaines JM, Huynh TN, Metter EJ. A home-based pedometerdriven walking program to increase physical activity in older adults with osteoarthritis of the knee: a preliminary study. J Am Geriatr Soc. 2003:51(3):387-92.

\section{Publisher's Note}

Springer Nature remains neutral with regard to jurisdictional claims in published maps and institutional affiliations.

Ready to submit your research? Choose BMC and benefit from:

- fast, convenient online submission

- thorough peer review by experienced researchers in your field

- rapid publication on acceptance

- support for research data, including large and complex data types

- gold Open Access which fosters wider collaboration and increased citations

- maximum visibility for your research: over $100 \mathrm{M}$ website views per year

At BMC, research is always in progress.

Learn more biomedcentral.com/submissions 